\title{
Obliterative Airway Remodeling
}

\section{Molecular Evidence for Shared Pathways in Transplanted and Native Lungs}

\author{
Danny Jonigk, ${ }^{*}$ Marlene Merk, ${ }^{*}$ Kais Hussein, ${ }^{*}$ \\ Lavinia Maegel, ${ }^{*}$ Katharina Theophile, ${ }^{*}$ \\ Michaela Muth, ${ }^{*}$ Ulrich Lehmann, ${ }^{*}$ \\ Clemens L. Bockmeyer, ${ }^{*}$ Michael Mengel, ${ }^{\S}$ \\ Jens Gottlieb, ${ }^{\dagger}$ Tobias Welte, ${ }^{\dagger}$ Axel Haverich, ${ }^{\ddagger}$ \\ Heiko Golpon, ${ }^{\dagger}$ Hans Kreipe, ${ }^{*}$ \\ and Florian Laenger*

\begin{abstract}
From the Institute of Pathology* and the Departments of Pneumology ${ }^{\dagger}$ and Thoracic Surgery, ${ }^{\ddagger}$ Hannover Medical School, Hannover, Germany; and the Department of Laboratory Medicine and Pathology, ${ }^{\S}$ University of Alberta Hospital, Alberta Health Services, Edmonton, Alberta, Canada
\end{abstract}

Obliteration of the small airways is a largely unresolved challenge in pulmonary medicine. It represents either the irreversible cause of functional impairment or a morphologic disorder of limited importance in a multitude of diseases. Bronchiolitis obliterans is a key complication of lung transplantation. No predictive markers for the onset of obliterative remodeling are currently available. To further elucidate the molecular mechanisms of airway remodeling, compartment-specific expression patterns were analyzed in patients. For this purpose, remodeled and nonremodeled bronchioli were isolated from transplanted and nontransplanted lung explants using laser-assisted microdissection $(n=24)$. mRNA expression of 45 fibrosis-associated genes was measured using quantitative real-time RT-PCR. For 20 genes, protein expression was also analyzed by immunohistochemistry. Infiltrating cells were characterized at conventional histology and immunohistochemistry. Obliterative remodeling of the small airways in transplanted and nontransplanted lungs shared similar grades of chronic inflammation and pivotal fibrotic pathways such as transforming growth factor $\beta$ signaling and increased collagen expression. Bone morphogenetic protein and thrombospondin signaling, and also matrix metalloproteinases and tissue inhibitor of metalloproteinases, were primarily up-regulated in obliterative airway remodeling in nontransplanted lungs. In transplanted lungs, clinical remodeled bone morphogenetic protein but nonremodeled bronchioli were characterized by a concordant up-regulation of matrix metalloproteinase-9, RANTES, and tissue inhibitor of metalloproteinase-1. These distinct expression patterns warrant further investigation as potential markers of impending airway remodeling, especially for prospective longitudinal molecular profiling. ( $\mathrm{AmJ}$ Pathol 2011, 178:599-608; DOI: 10.1016/j.ajpath.2010.10.032)

The histologic term "bronchiolitis obliterans" describes obliterative changes in the airways that commonly occur in a variety of pulmonary diseases. These changes demonstrate divergent histologic and radiologic findings, various potencies to progress to additional compartments of the lung, and different clinical outcomes. ${ }^{1,2}$ At histologic analysis, two major variants can be distinguished. The first is varying degrees of discontinuous submucosal collagenous deposits that narrow the lumen of the bronchioli up to the point of total obliteration, referred to as "constrictive bronchiolitis." This type is most commonly observed after hematopoietic stem cell transplantation or allogeneic lung transplantation, in which it significantly limits long-term survival and is considered the hallmark of chronic graft dysfunction. ${ }^{3}$ The second variant is bronchiolitis obliterans with intraluminal polyps, in which mesenchymal protrusions bulge into the lumen of the airways and the adjacent alveoli. This type is referred to as "organizing pneumonia" (formerly

Supported by grant DFG, KFO 123 LTx (D.J. and H.K.) from the "Deutsche Forschungsgemeinschaft"; and by the "Integriertes Forschungs und Behandlungszentrum Transplantation" (reference 01EO0802) from the German Federal Ministry of Education.

Accepted for publication October 21, 2010.

Supplemental material for this article can be found at $h$ ttp://ajp. amjpathol.org and at doi:10.1016/j.ajpath.2010.10.032.

Address reprint requests to Danny Jonigk, M.D., Institute of Pathology, Hannover Medical School, Carl-Neuberg-Str 1, D-30625 Hannover, Germany. E-mail: jonigk.danny@mh-hannover.de. 
"bronchiolitis obliterans organizing pneumonia"), and represents a nonspecific pattern. If a specific cause can be excluded clinically, the disorder is termed "cryptogenic organizing pneumonia." In most cases, cryptogenic organizing pneumonia responds well to steroid therapy, leading to rapid resolution of pulmonary symptoms and radiologic signs. In a small percentage of patients, however, organizing pneumonia does not respond to anti-inflammatory therapy, and continuous respiratory failure develops, with lung transplantation the only therapeutic option. ${ }^{4,5}$ To date, no differentiating markers for onset or progression of obliterative airway remodeling have been established.

In all of these entities and patterns, the small bronchioli represent the focus of (initial) activity, and (myo)fibroblasts contribute to the remodeling process by secreting profibrotic cytokines and producing abundant extracellular matrix (ECM). ${ }^{6}$ While (myo)fibroblasts have been demonstrated to descend from activated local mesenchymal cells or to transdifferentiate from respiratory epithelium through epithelial-mesenchymal transition, substantial quantities of these cells derive from the bone marrow of transplant recipients. ${ }^{7}$ While the definite mechanisms that lead to remodeling and organ dysfunction are not fully understood, it is accepted that induction and sustainment of fibrogenesis is regulated by a highly complex network of mediators, with the transforming growth factor- $\beta$ (TGF- $\beta$ ) cascade representing the lynchpin of remodeling. ${ }^{8-10}$ In addition to profibrotic mediators such as endothelin-1, lysil oxidase, and chemokine (C-C motif) ligand 5 [CCL5; synonym, RANTES (regulated on activation, normal T-cell expressed, and presumably secreted)], antifibrotic factors such as bone morphogenetic proteins (BMPs), matrix metalloproteinases (MMPs), and tissue inhibitors of metalloproteinase (TIMPs) contribute, at least in part, to the local microenvironment. ${ }^{11-15}$ These mediate fibrosis by degradation of ECM, influence hemostasis of fibroblasts, and activate other, in part opposing, cytokines that regulate fibrotic remodeling.

Obliterative remodeling usually originates in the small bronchioli. ${ }^{5}$ Inasmuch as routine transbronchial biopsy is insufficient to reproducibly detect lesions of bronchiolitis obliterans because of their discontinuous nature, and an ideal animal model for bronchiolitis obliterans has not yet been established, most of the earlier research was performed either using whole-tissue samples or in in vitro studies. In the present study, whole explanted lungs from patients who had or had not undergone lung transplantation were used for systematic analysis of airway obliteration in human beings, focusing on changes in the small bronchioli. Consequently, the nontransplantation group was quite heterogeneous, consisting primarily of patients with lung conditions that were nonresponsive to medication; otherwise, there would have been no need to undergo lung transplantation. ${ }^{4}$ Because primarily clinically defined entities such as neutrophilic reversible allograft dysfunction, which responds well to antibiotic therapy, continue to emerge, "obliterative airway remodeling" (OAR) is pro- posed as an umbrella term to encompass all structural changes in the small bronchioli. ${ }^{16,17}$ The objective of the present study was to further elucidate possible differences between the more or less distinct entities that make up OAR. Mediator profiles of obliterative remodeling in human lungs might point to possible markers of manifestation and aggravation and also to potential therapeutic targets, and would be of great value as an addendum to a morphologic diagnosis, especially in patients who have undergone lung transplantation.

\section{Materials and Methods}

\section{Specimens and Study Groups}

Twenty-four bilateral lung explants were analyzed. Twelve were explanted lung allografts with bronchiolitis obliterans. Mean (SD) patient age at transplantation was 39.3 (10.6) years, and time from transplantation to subsequent allograft explantation was 3.4 (2.2) years. Because explanted lung allografts can demonstrate a variety of histologic changes as sequelae of transplantation, inflammation, and infection, in addition to bronchiolitis obliterans sensu stricto, selected well-fixed cases were extensively sampled to confirm a predominant and "pure" bronchiolitis obliterans (constrictive bronchiolitis) pattern. These patients had received a macrolide-based antibiotic regimen as part of the treatment of bronchiolitis obliterans syndrome. Furthermore, 12 explanted native lungs with prominent nontransplantation-associated OAR were chosen [patient age at transplantation, 41.0 (16.1) years]. Underlying diseases that led to explantation included chronic organizing pneumonia, organizing pneumonia following inhalation trauma due to massive ammonia exposure in an occupational injury, bronchiolitis obliterans as a sequela of graft-versus-host disease after hematopoi-

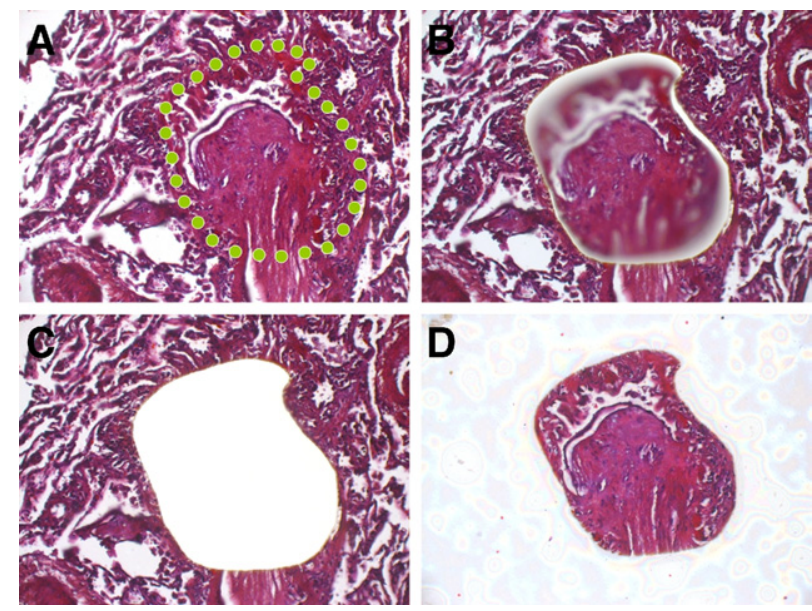

Figure 1. A-D: Laser-assisted microdissection (IX 71 microscope; Olympus Europa GmbH, Hamburg, Germany, with CellCut Plus system, MMI Molecular Machines \& Industries AG, Glattbrugg, Switzerland) was used to isolate an obliterated bronchiolus from the surrounding lung parenchyma. A: Border of excision is indicated by the green dotted line. B: Laser cut is visible. C: Microdissected area. D: Isolated obliterative mesenchymal plug is captured in the cap. H\&E was used to clarify the cellular composition. Hemalum staining was used for all samples from which mRNA was extracted. Original magnification, $\times 100$ 

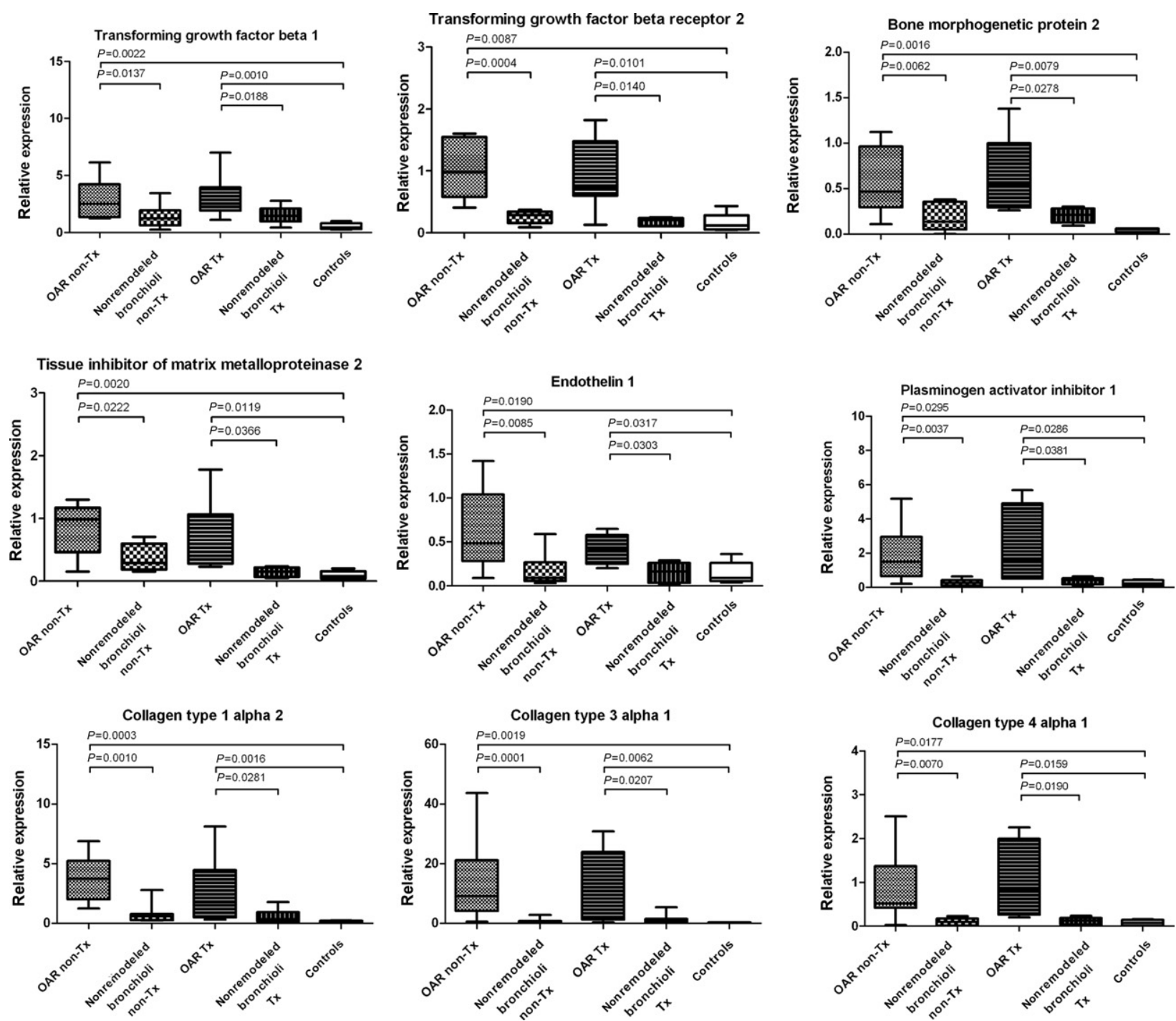

Figure 2. Shared mRNA expression characteristics in nontransplanted (non-Tx) and transplanted (Tx) lungs. Note significant up-regulation of TGF- $\beta 1$, TGFBR2, $B M P-2, T I M P-2, M M P-2, E D N 1, P L A 1, C O L 1 / \alpha 2, C O L 3 / \alpha 1$, and $C O L 4 / \alpha 1$ in OAR, compared with corresponding nonremodeled bronchioli from the same lung explants (Tx and non-Tx) and controls

etic stem cell transplantation, radiation-induced airway fibrosis following treatment of Hodgkin's Iymphoma, and idiopathic pulmonary fibrosis (IPF) with dominant OAR (see Supplemental Table S1 at http://ajp.amjpathol.org). In all cases, the OAR structure in the individual subgroups was matched as closely as possible.

Transbronchial biopsy specimens were analyzed from four patients who developed transplantation-associated OAR or bronchiolitis obliterans syndrome soon after transplantation and, thus, underwent a second transplantation procedure [mean (SD) age at transplantation, 29 (8) years, and time from initial transplantation to sampling of the examined biopsy specimens, 306 (155) days] and from four other patients who exhibited above-average survival free of bronchiolitis obliterans syndrome [age at transplantation, 39 (10) years, and time from initial transplantation to sampling of the examined biopsy specimens, 2335 (374) days]. The reference material was small airways from downsizing samples from donor lungs, which were resected just before lung transplantation $(n=5)$. The FFPE tissue samples were retrieved from the archives of the Institute of Pathology, Hannover Medical School (Hannover, Germany) and were handled anonymously according to the requirements of the local ethics committee.

\section{Light Microscopy and Immunohistochemistry}

Chronic inflammation in OAR and the adjacent nonobliterated bronchioli was graded according to microscopic findings on a scale of 0 to 3 , as follows: 0 , no lymphohistiocytic infiltrate; 1, sparse discontinuous infiltrate of lymphohistiocytic cells in the airway mucosa; 2, circumferential band of lymphocytes or histiocytes; and 3 , broad circumferential band of lymphocytes or histiocytes with epithelial inflammation or necrosis. Serially cut sections were immunohistochemically stained for different inflammation- and fibrosis-associated targets using commercially available antibodies and a 
Table 1. Comparison of mRNA Expression in OAR Non-Tx and OAR Tx Versus Adjacent Nonremodeled Bronchioli

\begin{tabular}{|c|c|c|}
\hline \multirow[b]{2}{*}{ Gene } & \multicolumn{2}{|c|}{ OAR } \\
\hline & Non-Tx recipients & Tx recipients \\
\hline TGF- $\beta 1$ & $P=0.0137^{\star}$ & $P=0.0188^{*}$ \\
\hline TGFBR2 & $P=0.0004^{\dagger}$ & $P=0.014^{\star}$ \\
\hline$B M P-2$ & $P=0.0062^{*}$ & $P=0.0278^{*}$ \\
\hline$B M P-4$ & $P=0.009^{\mp}$ & NS \\
\hline BMPR2 & $P=0.0066^{\ddagger}$ & NS \\
\hline MMP-14 & $P=0.0054^{\ddagger}$ & NS \\
\hline TIMP-1 & $P=0.0068^{\ddagger}$ & NS \\
\hline TIMP-2 & $P=0.0222^{*}$ & $P=0.0366^{\star}$ \\
\hline PLAU & $P=0.014^{\star}$ & NS \\
\hline PLA1 & $P=0.0037^{\ddagger}$ & $P=0.0381^{*}$ \\
\hline THBS1 & $P=0.001^{\ddagger}$ & NS \\
\hline$E D N 1$ & $P=0.0085^{\ddagger}$ & $P=0.0303^{*}$ \\
\hline LOX & $P=0.0034^{\ddagger}$ & NS \\
\hline RANTES & $P=0.0245^{\star}$ & NS \\
\hline $\operatorname{COL~} 1 / \alpha 2$ & $P=0.001^{\dagger}$ & $P=0.0281$ \\
\hline $\mathrm{COL} 3 / \alpha 1$ & $P=0.0001^{\dagger}$ & $P=0.0207$ \\
\hline $\mathrm{COL} 4 / \alpha 1$ & $P=0.007^{\ddagger}$ & $P=0.019^{\star}$ \\
\hline
\end{tabular}

Comparison of gene up-regulation in obliterative airway remodeling in nontransplant and transplant recipients. Adjacent nonremodeled bronchioli from the same lungs were used as endogenous controls.

NS, not significant; OAR, obliterative airway remodeling; Tx, transplantation.

${ }^{*} P<0.05$

$+P<0.001$

$\ddagger P<0.01$.

standard ABC protocol (see Supplemental Table S2 at http://ajp.amjpathol.org). Staining of the obliterated and nonobliterated small airways was scored semiquantitatively on a scale of 0 to 3 , as follows: 0 , no apparent reaction; 1 , positivity in less than $30 \%$ of cells; 2 , positivity in more than $30 \%$ and less than $60 \%$ of cells; and 3 , positivity in more than $60 \%$ of cells. Staining intensity was graded as absent, weak, intermediate, or strong. For negative controls, the primary antibody was replaced with bovine serum albumin.

\section{Laser-Assisted Microdissection and RNA Extraction}

FFPE tissue sections $5 \mu \mathrm{m}$ thick were mounted on a polyL-lysin-coated membrane attached to a metal frame. After routine deparaffinization and hemalum staining, laser-assisted microdissection of target structures was performed using the CellCut Plus system (MMI Molecular Machines \& Industries AG, Glattbrugg, Switzerland). Obliterated and nonobliterated bronchioli from the same explants and unaltered bronchioli from the reference lung samples were isolated using a no-touch technique, as previously described (Figure 1). ${ }^{18}$ The respective compartments were sampled from different locations in both lobes of the explants. They were morphologically chosen to adequately reflect the average degree of fibrotic obliteration and cellular or inflammatory infiltrate in both OAR and nonobliterated bronchioli. Nonobliterated bronchioli were defined as small airways without delimitable metaplasia of the epithelium, significant thickening of the basal lamina, or fibrosis.

Approximately 8500 cells were harvested from serial sections in each compartment. The microdissected tissue was subsequently suspended in a proteinase $\mathrm{K}$ di- gestion buffer by placing the buffer directly in the adhesive cap. After overnight digestion, RNA was isolated using phenol-chloroform extraction and precipitation via established procedure. ${ }^{19}$

\section{cDNA Synthesis}

cDNA of each sample was generated from $1 \mu \mathrm{g}$ of RNA using a kit (High Capacity cDNA Reverse Transcription Kit; Applied Biosystems, Inc., Foster City, CA) and according to the manufacturer's protocol.

\section{Low-Density Arrays}

Forty-five target genes and three reference genes were selected for a custom-made array (TaqMan Low-Density Array; Applied Biosystems, Inc.). For genes and abbreviations, see Supplemental Table S3 at http://ajp.amjpathol. org. An amplicon size less than $100 \mathrm{bp}$ was one of the criteria for target genes, enabling reliable gene expression analysis using a low-density array in FFPE samples. The primer sets were spotted eightfold $(8 \times 48)$ into a 384-well plate, enabling synchronous analysis of eight samples per PCR run. The low-density arrays were performed as single runs using a $7900 \mathrm{HT}$ Fast Real-Time PCR system, and recorded using 7900HT SDS 2.3 software (Applied Biosystems, Inc.). For negative controls, cDNA was replaced with water. ${ }^{20}$

\section{Statistical Analysis and Graphics}

In the various study groups, cycle threshold values were established via normalization to the mean expression of the three endogenous controls. ${ }^{21}$ One-way analysis of variance was performed, followed by Dennett's multiple comparison tests. $P \leq 0.05$ was considered statistically significant. Expression graphics were created using commercially available software (GraphPad Prism, version 5.0; GraphPad Software, Inc., San Diego, CA).

\section{Results}

\section{Shared Expression Characteristics in OAR in Transplant and Nontransplant Recipients}

Anatomically defined compartments were analyzed using laser-assisted microdissection to generate specific quantitative mRNA expression profiles of the pathologically affected structures and to correlate them with the results of immunohistochemistry. Microdissected obliterated bronchioli comprised smooth muscle cells, ECM, (myo)fibroblasts, infiltrating leukocytes, and remnant epithelium. Naïve nonremodeled bronchioli demonstrated the physiologic composition of smooth muscle cells, ECM, epithelium, and leukocytes. 

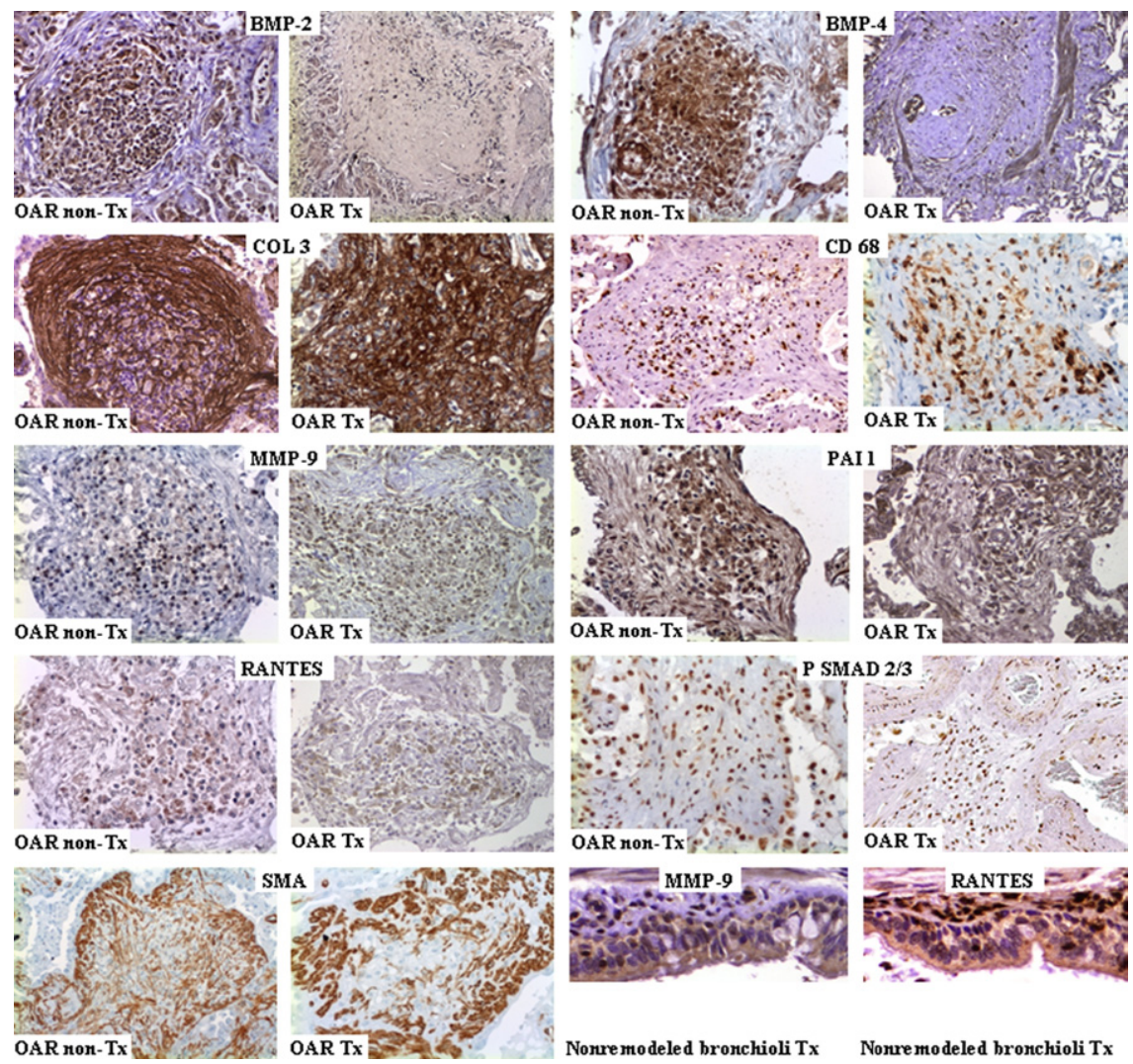

Nonre modeled bronchioli $\mathrm{T} x$

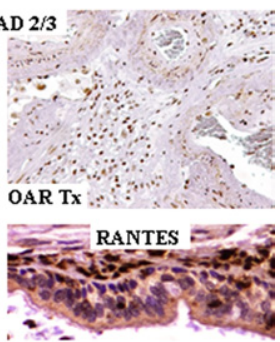

Nonre modeled bronchioli $\mathrm{T} \times$
Figure 3. Protein expression of fibrosis- and in flammation-associated markers in OAR in nontransplanted and transplanted lung specimens (OAR non-Tx and OAR Tx): BMP-2 shows strong cytoplasmic positivity in infiltrating inflammatory and mesenchymal cells. BMP-4 shows cytoplasmic positivity in mesenchymal cells and remnant respiratory epithelium. Fibroblastic cells show marked membranous and extracellular positivity for COL 3 . A significant number of local inflammatory cells are $\mathrm{CD} 68^{+}$macrophages. MMP-9, plasminogen activator inhibitor-1 (PAI1), and RANTES stain positive in the cytoplasm of infiltrating inflammatory and mesenchymal cells. Phospho (P)-SMAD 2/3 exhibits strong positivity in the nuclei of inflammatory and both mesenchymal and fibroblastic cells. Fibroblastic cells demonstrate marked membranous and extracellular positivity for smooth muscle actin (SMA). Note the MMP-9- and RANTES-positive infiltrating cells in the nonremodeled bronchioli in lung transplant recipients. Original magnification: OAR Tx/BMP-2 and BMP-4, $\times 25$; OAR Tx/MMP-9 and RANTES, $\times$ 400; all other images, $\times 200$ ).
Remodeled bronchioli in transplant and nontransplant recipients shared significant $\mathrm{mRNA}$ up-regulation of TGF31, TGFBR 2, BMP-2, TIMP-2, MMP-2, EDN1, PLA1, and $\mathrm{COL} 1 / \alpha 2,3 / \alpha 1$, and $4 / \alpha 1$, compared with nonremodeled bronchioli from the same explants and controls (Figure 2,
Table 1). This concurs with the complementary immunohistochemistry for TGF- $\beta 1$, BMP-2, and collagen type 3, which likewise exhibited the strongest positivity in the mesenchymal cells and the remaining respiratory epithelium (not collagen type 3) of the obliterated bronchioli (Figure 3, Table 2).

Table 2. Results of Immunohistochemical Staining*

\begin{tabular}{|c|c|c|c|c|}
\hline Protein & OAR non-Tx & OAR Tx & $\begin{array}{c}\text { Nonremodeled } \\
\text { bronchioli, non-Tx }\end{array}$ & $\begin{array}{r}\text { Nonremodeled } \\
\text { bronchioli, Tx }\end{array}$ \\
\hline RANTES/CCL-5 & 2/Intermediate & 3/Strong & 1/Intermediate & 2/Strong \\
\hline PAl1 & 3/Strong & 3/Strong & $1 /$ Intermediate & 1/Weak \\
\hline SMAD1 & 1/Intermediate & $1 /$ Intermediate & 1/Weak & 1/Weak \\
\hline SMAD2 & 2/Intermediate & 2/Weak & 1/Weak & 1/Weak \\
\hline SMAD3 & 1/Weak & 1/Weak & O/Absent & O/Absent \\
\hline Phospho-SMAD 1/5 & 2/Weak & 1/Weak & 1/Weak & 1/Weak \\
\hline Phospho-SMAD 2/3 & 3/Strong & 3/Strong & 1/Weak & 1/Weak \\
\hline TGF- $\beta 1$ & 3/Strong & 2/Strong & 1/Weak & 1/Weak \\
\hline BMP-1 & 1/Weak & 2/Weak & 1/Weak & 1/Weak \\
\hline BMP-2 & 2/Strong & 3/Strong & 1/Weak & 1/Weak \\
\hline BMP-4 & 3/Strong & 2/Strong & 1/Weak & 1/Weak \\
\hline BMP-7 & $1 /$ Intermediate & 3/Strong & 1/Weak & 1/Weak \\
\hline MMP-9 & 1/Intermediate & 2/Strong & 1/Weak & 2/Strong \\
\hline Collagen 3 & 3/Strong & 3/Strong & O/Absent & O/Absent \\
\hline Smooth muscle actin & 3/Strong & 3/Strong & O/Absent & O/Absent \\
\hline $\begin{array}{l}\text { Smooth muscle myosin } \\
\text { heavy chain }\end{array}$ & 3/Strong & 2/Strong & O/Absent & O/Absent \\
\hline Caldesmon & 1/Weak & 1/Weak & O/Absent & O/Absent \\
\hline Desmin & 1/Intermediate & $1 /$ Intermediate & O/Absent & O/Absent \\
\hline CD 45 & 2/Strong & 1/Strong & $1 /$ Intermediate & $1 /$ Intermediate \\
\hline CD 68 & 2/Strong & 2/Strong & $1 /$ Intermediate & 2/Intermediate \\
\hline
\end{tabular}

OAR, obliterative airway remodeling; $\mathrm{Tx}$, transplantation

*Data are given as range of positive cells ( 0 , no apparent reaction; 1 , positivity in $<30 \%$ of cells; 2 , positivity in $>30 \%$ but $<60 \%$ of cells; and 3 , positivity in $>60 \%$ of cells) and staining intensity. 

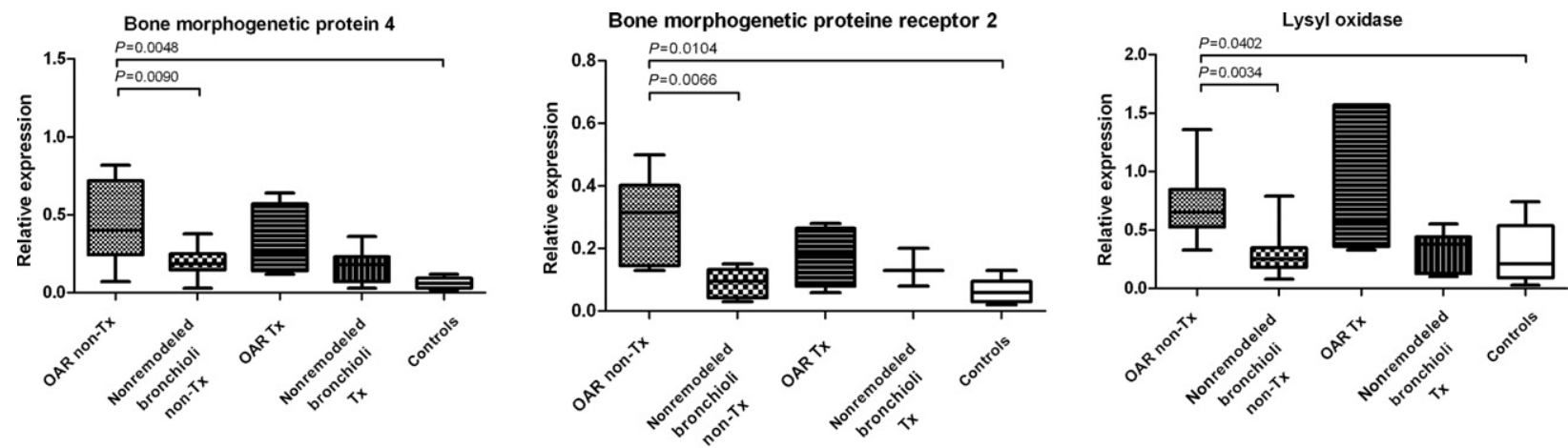

Matrix metalloproteinase 14 (membrane-type MMP 1)
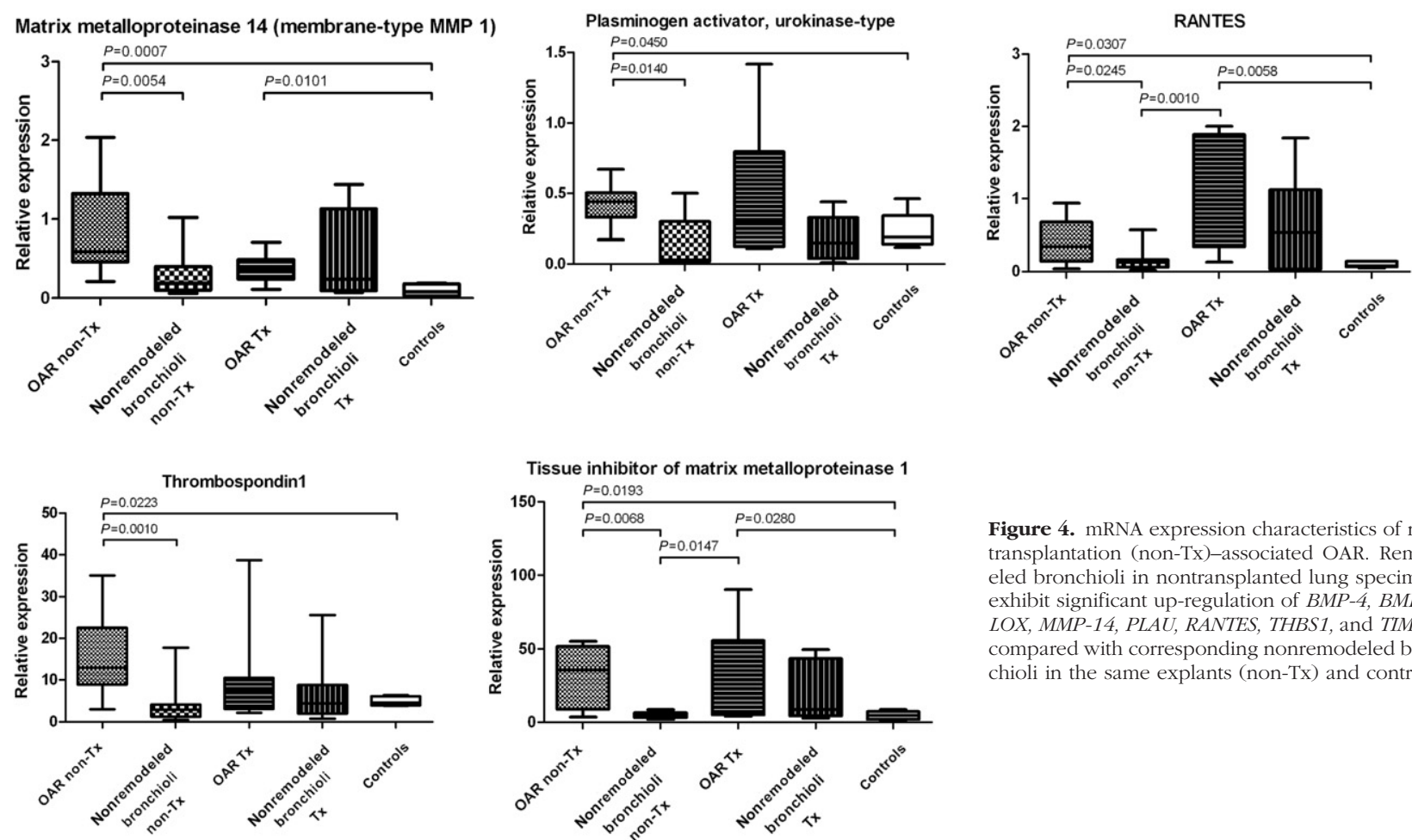

Figure 4. mRNA expression characteristics of nontransplantation (non-Tx)-associated OAR. Remodeled bronchioli in nontransplanted lung specimens exhibit significant up-regulation of $B M P-4, B M P R 2$, LOX, MMP-14, PLAU, RANTES, THBS1, and TIMP-1, compared with corresponding nonremodeled bronchioli in the same explants (non-Tx) and controls.

\section{Differential Characterization of Nontransplantation-Associated OAR}

Remodeled bronchioli in nontransplanted but not transplanted lung specimens demonstrated mRNA up-regulation of BMP-4, BMPR2, LOX, MMP-14, PLAU (plasminogen activator, urokinase), RANTES, THBS1, and TIMP-1, compared with nonremodeled bronchioli in the same explants and in controls. The complementary immunohistochemistry for BMP-4 and RANTES was positive in the mesenchymal cells composing the fibrous obliteration and the scarce remaining respiratory epithelium (Figures 3 and 4 ; Tables 1 and 2).

\section{Characterization of Subgroups in Nontransplantation-Associated OAR}

In the largest subgroup of nontransplant recipients with OAR (IPF/OAR), significant up-regulation of MMP-14 and
THBS1 was observed, compared with endogenous controls (nonremodeled bronchioli IPF/OAR), as well as obliterative airway remodeling in transplant recipients. A trend toward higher levels of expression of TGF- $\beta 1$ was observed in patients with IPF/OAR compared with transplantation-associated OAR. Compared with the second largest subgroup of nontransplant recipients with OAR (due to graft-versus-host disease), IPF/OAR demonstrated significant up-regulation of TGF- $\beta 1$, with a trend toward up-regulation of MMP-14 $(P=0.0714)$ and THBS1 $(P=0.099)$. The smaller subgroups in the nontransplanted arm of the study did not demonstrate significant differences in expression (Figures 3 and 5).

\section{Characterization of Transplantation-Associated OAR According to MMP-9, RANTES, and TIMP-1}

Both OAR and nonremodeled bronchioli in explanted lung allografts demonstrated a concordant increase in 


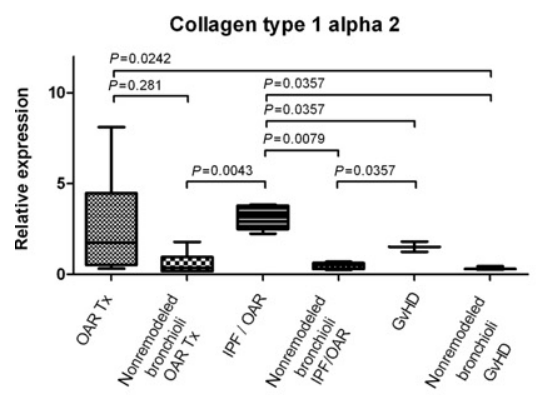

Matrixmetalloproteinase 14 (membrane-type MMP1)
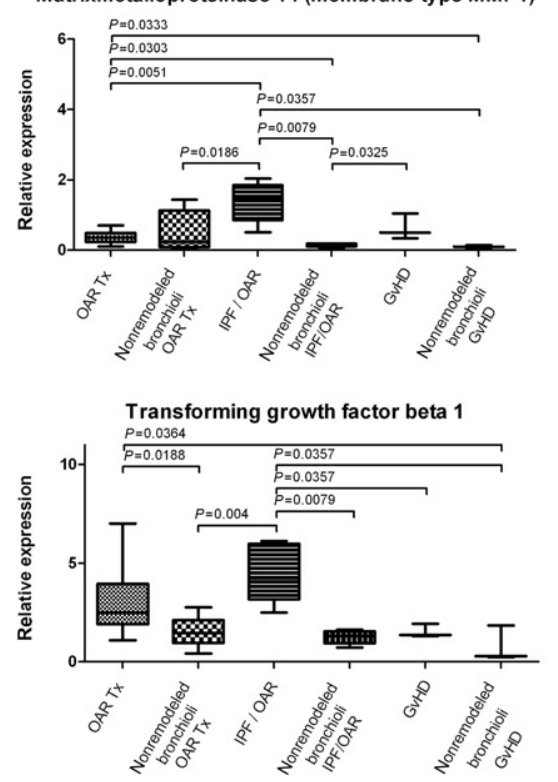
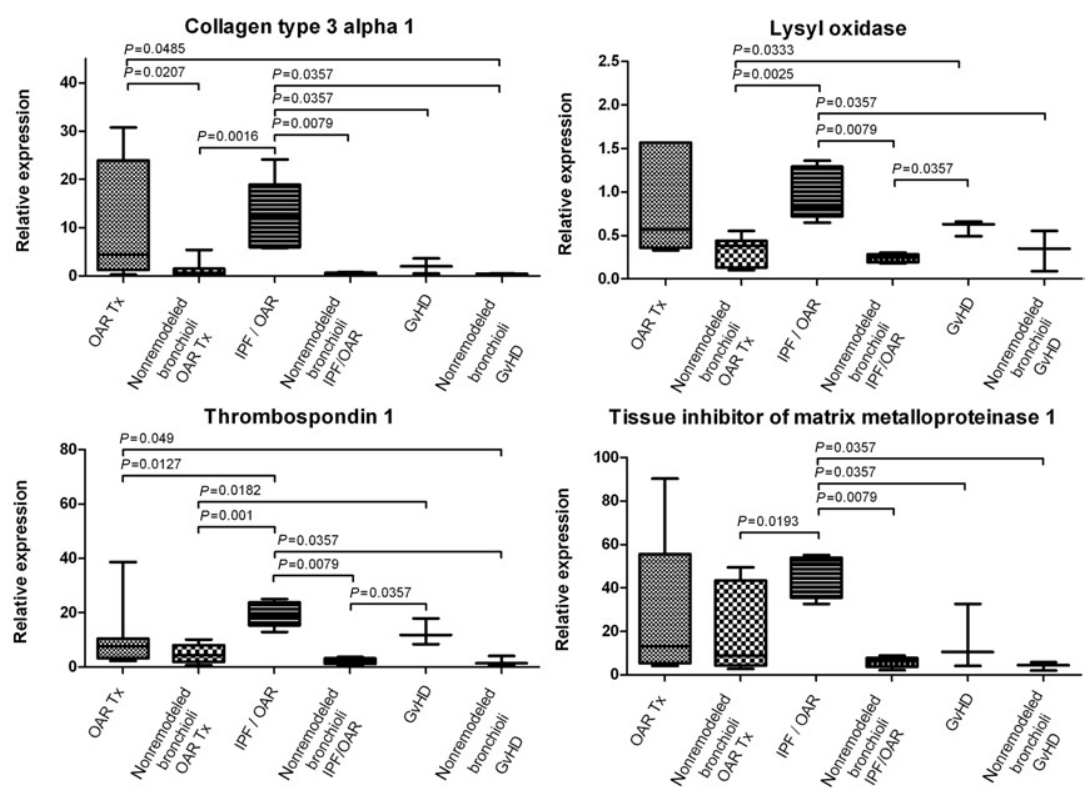

Tissue inhibitor of matrix metalloproteinase 1

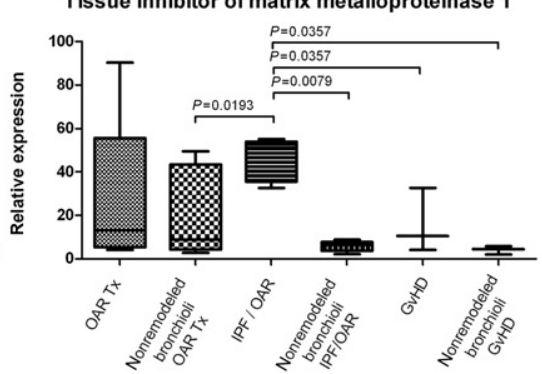

Figure 5. Selected mRNA expression characteristics of subgroups in nontransplantation (non-Tx) associated OAR. Remodeled bronchioli in lungs with interstitial pulmonary fibrosis with distinctive obliterative airway remodeling (IPF/OAR) exhibit significant up-regulation of $M M P-14$ and THBS1, compared with endogenous controls (nonremodeled bronchioli IPF/OAR), and to obliterative airway remodeling in transplant recipients (OAR Tx). TGF- $\beta 1$ shows a trend toward higher levels of expression in IPF/OAR than in OAR Tx. Compared with the nontransplant subgroup of patients with OAR due to graft-versus-host disease (GvHD), IPF/OAR exhibited significant up-regulation of $M M P-14$ and TGF- $\beta 1$, with a trend toward THBS1 up-regulation. Collagens (COL) in OAR in different subgroups also exhibit significant up-regulation, compared with nonremodeled bronchioli (Tx and non-Tx).

mRNA expression of MMP-9, RANTES, and TIMP-1, compared with remodeled and nonremodeled bronchioli from nontransplanted lungs and controls. The immunohistochemistry staining was observed to be compatible, with strong positivity in both compartments in the grafts (Figure 6, Table 2).

\section{Consistency of mRNA Expression in OAR and Nonremodeled Bronchioli}

To verify that no major variation existed between samples from different locations in the same explants, three explanted allografts with OAR and isolated obliterated and nonremodeled bronchioli from the upper and lower lobe in each lung ( $n=4$ in each explant) were selected (see Materials and Methods). In all of these samples, mRNA expression of MMP-9, RANTES, and TIMP-1 using real-time PCR, subsequent to gene-specific preamplification, were analyzed as previously described. ${ }^{19}$ The variation of mRNA expression of different sites within the individual lungs was minimal and not significant $(P>0.05)$ (Figure S1).

\section{Evaluation of End-Stage Markers in Transbronchial Biopsy Specimens}

The concept of concordant MMP-9, RANTES, and TIMP-1 up-regulation as an end-stage marker was proved using transbronchial biopsy specimens from 4 patients who developed transplantation-associated OAR or bronchiolitis obliterans syndrome soon after transplantation, and 4 patients who exhibited above-average survival free of bronchiolitis obliterans syndrome. Laser-assisted microdissection was performed to isolate the nonremodeled bronchioli from each biopsy specimen. In all of these samples, mRNA expression of MMP-9, RANTES, and TIMP-1 was analyzed using real-time PCR, as described. Significant mRNA up-regulation of RANTES and TIMP-1 was observed in nonremodeled bronchioli in patients with rapid-onset OAR compared with nonremodeled bronchioli from biopsy specimens from patients free of bronchiolitis obliterans syndrome. MMP-9 demonstrated a trend toward up-regulation in nonremodeled bronchioli in patients with rapid-onset OAR $(P=0.058)$ (Figure 7$)$.

\section{Increased Immunohistochemical Staining Pattern for Phospho-SMAD in OAR}

Although mRNA expression did not yield significant differences in expression of SMAD1, SMAD3, SMAD4, and SMAD5 (Sma- and Mad-related proteins) between the various anatomical compartments, nuclear staining of phospho-SMAD 2/3 was strongest in remodeled airways in transplanted and nontransplanted lungs com- 

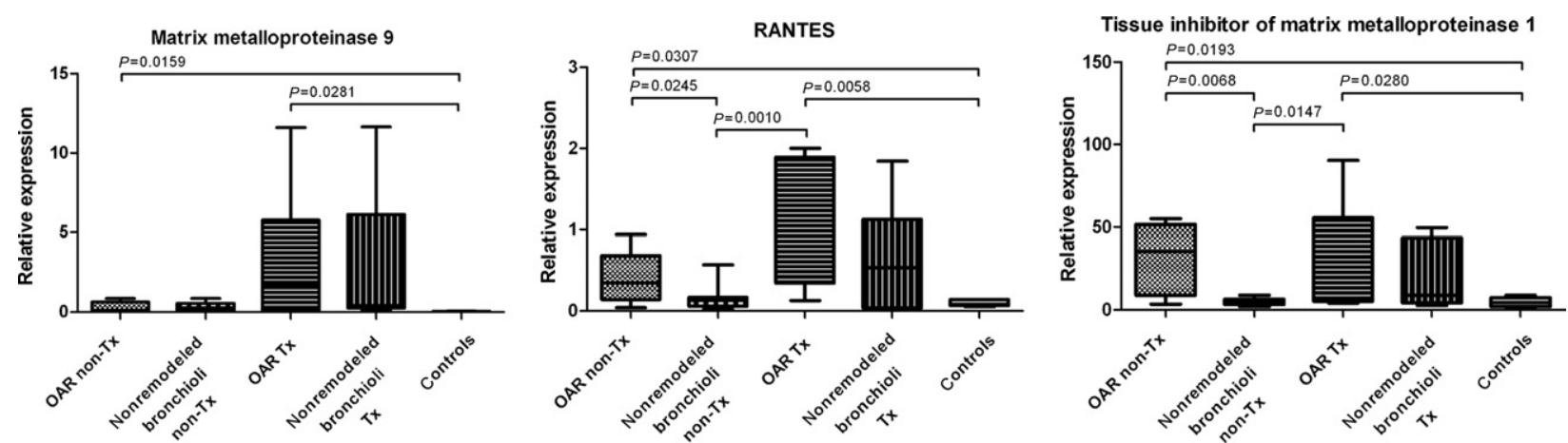

Figure 6. Concordant mRNA expression of MMP-9, RANTES, and TIMP-1 characterizes allografts with OAR (Tx). Both obliterated and nonremodeled bronchioli from explanted lung allografts demonstrate concordant up-regulation of $M M P-9, R A N T E S$, and TIMP-1, compared with nonremodeled bronchioli from nontransplanted (non-Tx) lungs and controls.

pared with endogenous controls (native bronchioli in the same organs) (Figure 3, Table 2).

\section{Cellularity of OAR in Transplanted and Nontransplanted Explants}

Overall, remodeled bronchioli in transplanted and nontransplanted explants demonstrated significantly more infiltrating inflammatory cells compared with patent bronchioli $(P<0.01)$ (Table 3). CD 68-positive macrophages were significantly increased in OAR (obliterated airways mean, $\leq 60 \%$ of infiltrating leukocytes positive for CD 68; $P=0.0005$; Figure 3; Table 2). The same was true for myofibroblasts, which were identified by architecture and expression of smooth muscle actin (obliterated airways, $>60 \%$ of mesenchymal cells positive for smooth muscle actin; nonremodeled bronchioli mean, $\leq 30 \%$ positive for smooth muscle actin; $P$ $<0.0001$ ). OAR sensu stricto (excluding peribronchial fibroblastic foci or comparable hallmarks of IPF in nontransplanted specimens) did not differ significantly insofar as cellularity or cellular composition between transplant and nontransplant recipients $(P>0.05$; Table 3$)$. Overall, in the transplantation group of our collective, the time from initial implantation to graft explantation did not have a significant influence on cellularity or cellular composition of OAR $(P>0.05)$.

\section{Discussion}

OAR in the human lung represents a largely unsolved challenge in pulmonary medicine, in particular after lung transplantation. Recent years have witnessed a breakthrough in management of acute cellular rejection and chronic allograft dysfunction, and its main morphologic hallmark, bronchiolitis obliterans, has emerged as a major complication and the leading cause of death. ${ }^{3}$

The outcome in patients with OAR who did not undergo lung transplantation varies significantly and depends first and foremost on the underlying setting or disease and, thereby, on the responsiveness to medication (eg, steroid therapy). The involvement of a large number of effector molecules and their corresponding receptors in OAR has been demonstrated recently; however, the general inaccessibility of the local remodeling process by sampling at bronchoscopy has hampered adequate molecular profiling of the lesions. In addition, an ideal animal model of OAR that would enable sequential sacrificing and analysis of animals as remodeling progresses is yet to be established. ${ }^{22,23}$

At histologic analysis, the cohort of transplanted and nontransplanted lungs with OAR did not demonstrate major differences insofar as cellularity or chronic inflammation in obliterative remodeling of the small airways.
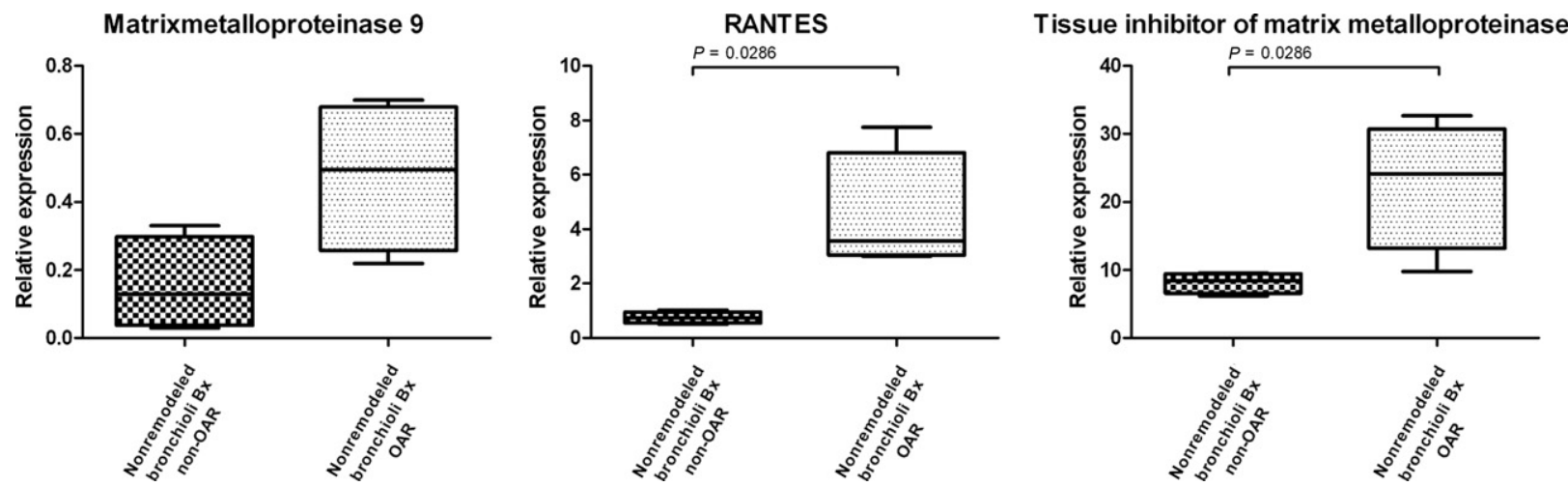

Figure 7. Up-regulation of mRNA expression of MMP-9, RANTES, and TIMP-1 in nonremodeled bronchioli from transbronchial allograft biopsy specimens from patients with rapid-onset bronchiolitis obliterans syndrome (nonremodeled bronchioli Bx OAR), compared with nonremodeled bronchioli from transbronchial allograft biopsy specimens from long-term survivors free of bronchiolitis obliterans syndrome (nonremodeled bronchioli Bx non-OAR). 
Table 3. Inflammation in Small Airways

\begin{tabular}{lccc}
\hline \multicolumn{1}{c}{ Variable } & & \multicolumn{2}{c}{ Grade of inflammation* } \\
\cline { 3 - 4 } & No. of patients & Obliterated bronchioli & Nonremodeled bronchioli \\
\hline Lung allograft & 12 & 2 & 1 \\
Interstitial pulmonary fibrosis & 5 & 2 & 1 \\
Graft-versus-host disease & 3 & 2 & 1 \\
Cryptogenic organizing pneumonia & 1 & 1 & 1 \\
Occupational injury (NH $\mathrm{NH}_{3}$ exposure) & 1 & 2 & 1 \\
Radiation-induced fibrosis from treatment of & 1 & 2 & 2 \\
Hodgkin's disease & 1 & 2 & 2 \\
Severe bronchiectasis & & 2 \\
\hline
\end{tabular}

*Score: 0 , no lymphohistiocytic infiltrate; 1 , singular lymphohistiocytic cells in airway mucosa; 2 , circumferential band of lymphocytes/histiocytes; 3 , broad circumferential band of lymphocytes/histiocytes with epithelial inflammation/necrosis.

The same was mostly true for the local microenvironment, which exhibited up-regulation of distinct enzymes and cytokines in both groups. Thus, findings of the present study point to a certain level of similarity between obliterative bronchiolitis in chronically rejected allografts and chronically failing nontransplanted lungs; they share overexpression of profibrotic cytokines such as endothelin-1, which have been found to be overexpressed in the interstitium of lungs with IPF and in cells and fluid obtained at bronchoalveolar lavage, which hints at its potential role as a biomarker for IPF, and also pivotal TGF- $\beta 1$ and TGF- $\beta R 2 .{ }^{11,24,25}$ TIMP-2 and BMP-2 expression was also elevated in the obliterated bronchioli in both groups. TIMP-2 inhibits MMPs and, thus, increases collagen accumulation, decreases reepithelialization of the airways, and facilitates epithelial-mesenchymal transition. ${ }^{26,27}$ Although the present study found that BMP-2 was elevated in remodeling of the bronchioli, others have previously demonstrated its involvement in smooth muscle cell proliferation in pulmonary hypertension. ${ }^{28}$ PLA1 was up-regulated in transplanted and nontransplanted lungs, as were COL-1, COL-2, and COL-3.

Other groups have confirmed these findings, and describe a consistent correlation of plasminogen activator inhibitor-1 and collagen expression and deposition in fibrosing lesions. ${ }^{29,30}$ The continuous and prominent expression of collagens, especially of collagen-3, that characterizes granulation tissue and "young" (myo)fibroblasts hints at ongoing, and potentially influenceable, dynamics of remodeling, even at the welladvanced state of the examined explants. MMP-2 expression was mutually increased farther downstream on the fibrosis cascade, where it is involved in maturation of $\mathrm{ECM}^{2}{ }^{2}$

Only OAR in nontransplanted lungs showed increased expression of BMP-4 and BMPR2. While originally named for their ability to facilitate osseous tissue formation, more recent experiments have demonstrated involvement of BMP signaling balance in lung development and fibrosis control of soft tissue turnover. While the link between BMP and TGF- $\beta$ is yet to be fully elucidated, various types of dynamic interaction such as shared receptors and competition for subordinate, in part inhibitory, SMADs have been described. ${ }^{31-33}$ All serve to maintain tissue homeostasis. Both increased and decreased levels of BMPs have been described in patients with fibrotic remodeling in different organs, and in vitro studies have attributed BMPs with a degree of protective antifibrotic properties. $^{34,35}$ The up-regulation of PAl1 and RANTES in nontransplanted specimens matches findings in patients with obstructive bronchitis, in whom it has been demonstrated to contribute to chronic inflammation. Like the BMPs, $P L A U$ is credited, at least in part, with antifibrotic properties because it also degrades the ECM. ${ }^{36}$ The increased expression of THBS1 matches findings of high concentrations in bronchoalveolar lavage cells and fluid from patients with fibrosing lung conditions. ${ }^{37}$ Furthermore, it activates MMPs. In the present study, increased expression of MMP-4 in OAR was observed in nontransplanted lungs, in which it presumably contributes to maturation of collagens, like lysil oxidase, while the also increased TIMP-1 hinders both MMPs and reepithelialization. ${ }^{38}$

MMP-9, RANTES, and TIMP-1 were prominently increased in both the remodeled and the nonremodeled morphologically inconspicuous bronchioli in transplant recipients. Whether these molecular events precede the morphologic changes or reflect a "field effect" from neighboring, already remodeled airways is unclear. Results of the present study do not explicitly define MMP-9, RANTES, and TIMP-1 as predictive markers for OAR. An animal model of bronchiolitis obliterans syndrome, providing the opportunity to study the changes at defined times, or a control group of explanted longterm functional allografts without OAR will be necessary. A suitable animal model has not yet been established, and the primary reason for a repeat lung transplantation procedure is chronic allograft dysfunction with morphologic features of OAR. Nevertheless, the concordant up-regulation of the above-mentioned genes in transbronchial biopsy specimens that exhibited no apparent airway remodeling but were from transplanted lungs with severe clinical bronchiolitis obliterans syndrome support the findings in explanted lungs. Therefore, concerted up-regulation of MMP-9, RANTES, and TIMP-1 is considered an "end-stage marker." Because routinely sampled transbronchial biopsy specimens lack adequate sensitivity for detecting the patchy discontinuous fibrotic plugs in the small airways that make up OAR, these genes represent possible targets for prospective longitudinal molecular profiling in the care of lung transplant recipients. 


\section{Acknowledgments}

We thank Gillian Teicke, Cord Albat, Ph.D., and Britta Hasemeier, M.S., for editing the text, and Henriette Christgen for technical assistance.

\section{References}

1. Bergeron C, Al-Ramli W, Hamid Q: Remodeling in asthma. Proc Am Thorac Soc 2009, 6:301-305

2. Sato M, Liu M, Anraku M, Ogura T, D'Cruz G, Alman BA, Waddell TK, Kim E, Zhang L, Keshavjee S: Allograft airway fibrosis in the pulmonary milieu: a disorder of tissue remodeling. Am J Transplant 2008, 8:517-528

3. Belperio JA, Weigt SS, Fishbein MC, Lynch JP III: Chronic lung allograft rejection: mechanisms and therapy. Proc Am Thorac Soc 2009, 6:108-121

4. Sara AG, Hamdan AJ, Hanaa B, Nawaz KA: Bronchiolitis obliterans organizing pneumonia: pathogenesis, clinical features, imaging and therapy review. Ann Thorac Med 2008, 3:67-75

5. Sato M, Keshavjee S: Bronchiolitis obliterans syndrome: alloimmunedependent and -independent injury with aberrant tissue remodeling. Semin Thorac Cardiovasc Surg 2008, 20:173-182

6. Eyden B: The myofibroblast: phenotypic characterization as a prerequisite to understanding its functions in translational medicine. J Cell Mol Med 2008, 12:22-37

7. Brocker V, Langer F, Fellous TG, Mengel M, Brittan M, Bredt M, Milde S, Welte T, Eder M, Haverich A, Alison MR, Kreipe H, Lehmann U: Fibroblasts of recipient origin contribute to bronchiolitis obliterans in human lung transplants. Am J Respir Crit Care Med 2006, 173:12761282

8. Pegorier S, Campbell GA, Kay AB, Lloyd CM: Bone morphogenetic protein (BMP)-4 and BMP-7 regulate differentially transforming growth factor (TGF)-beta1 in normal human lung fibroblasts (NHLF). Respir Res 2010, 11:85

9. Ward C, De Soyza A, Fisher AJ, Pritchard G, Forrest I, Corris P: A descriptive study of small airway reticular basement membrane thickening in clinically stable lung transplant recipients. J Heart Lung Transplant 2005, 24:533-537

10. Wynn TA: Cellular and molecular mechanisms of fibrosis. J Pathol 2008, 214:199-210

11. Swigris JJ, Brown KK: The role of endothelin- 1 in the pathogenesis of idiopathic pulmonary fibrosis 2010, BioDrugs 24:49-54

12. Chen LJ, Li WD, Li SF, Su XW, Lin GY, Huang YJ, Yan GM: Bleomycin induces upregulation of lysyl oxidase in cultured human fetal lung fibroblasts. Acta Pharmacol Sin 2010, 31:554-559

13. Kakizaki T, Kohno M, Watanabe M, Tajima A, Izumi Y, Miyasho T, Tasaka S, Fukunaga K, Maruyama I, Ishizaka A, Kobayashi K: Exacerbation of bleomycin-induced injury and fibrosis by pneumonectomy in the residual lung of mice. J Surg Res 2009, 154:336-344

14. Weiskirchen R, Meurer SK, Gressner OA, Herrmann J, BorkhamKamphorst E, Gressner AM: BMP-7 as antagonist of organ fibrosis. Front Biosci 2009, 14:4992-5012

15. Yamashita M, Yamauchi K, Chiba R, Iwama N, Date F, Shibata N, Kumagai H, Risteli J, Sato S, Takahashi T, Ono M: The definition of fibrogenic processes in fibroblastic foci of idiopathic pulmonary fibrosis based on morphometric quantification of extracellular matrices. Hum Pathol 2009, 40:1278-1287

16. Jonigk D, Theophile K, Hussein K, Bock O, Lehmann U, Bockmeyer CL, Gottlieb J, Fischer S, Simon A, Welte T, Maegel L, Kreipe H, Laenger F: Obliterative airway remodelling in transplanted and nontransplanted lungs. Virchows Arch 2010, 457:369-380

17. Verleden GM, Vos R, DE Vleeschauwer SI, Willems-Widyastuti A, Verleden SE, Dupont LJ, Van Raemdonck DE, Vanaudenaerde BM: Obliterative bronchiolitis following lung transplantation: from old to new concepts? Transpl Int 2009, 22:771-779

18. Jonigk D, Lehmann U, Stuht S, Wilhelmi M, Haverich A, Kreipe H, Mengel M: Recipient-derived neoangiogenesis of arterioles and lymphatics in quilty lesions of cardiac allografts. Transplantation 2007, 84:1335-1342
19. Theophile K, Jonigk D, Kreipe H, Bock O: Amplification of mRNA from laser-microdissected single or clustered cells in formalin-fixed and paraffin-embedded tissues for application in quantitative real-time PCR. Diagn Mol Pathol 2008, 17:101-106

20. Bock O, Muth M, Theophile K, Winter M, Hussein K, Busche G, Kroger $\mathrm{N}$, Kreipe $\mathrm{H}$ : Identification of new target molecules PTK2. TGFBR2 and CD9 overexpressed during advanced bone marrow remodelling in primary myelofibrosis. Br J Haematol 2009, 146:510-520

21. Livak KJ, Schmittgen TD: Analysis of relative gene expression data using real-time quantitative PCR and the 2(-Delta Delta $\mathrm{C}(\mathrm{T})$ ) method. Methods 2001, 25:402-408

22. Panoskaltsis-Mortari A, Tram KV, Price AP, Wendt CH, Blazar BR: A new murine model for bronchiolitis obliterans post-bone marrow transplant. Am J Respir Crit Care Med 2007, 176:713-723

23. Krupnick AS, Lin X, Li W, Okazaki M, Lai J, Sugimoto S, Richardson SB, Kornfeld CG, Garbow JR, Patterson GA, Gelman AE, Kreisel D: Orthotopic mouse lung transplantation as experimental methodology to study transplant and tumor biology. Nat Protoc 2009, 4:86-93

24. Chen H, Zhuang F, Liu YH, Xu B, Del Moral P, Deng W, Chai Y, Kolb M, Gauldie J, Warburton D, Moses HL, Shi W: TGF-beta receptor II in epithelia versus mesenchyme plays distinct roles in the developing lung. Eur Respir J 2008, 32:285-295

25. Ross B, D'Orleans-Juste P, Giaid A: Potential role of endothelin-1 in pulmonary fibrosis: from the bench to the clinic. Am J Respir Cell Mol Biol 2010, 42:16-20

26. Yang K, Palm J, Konig J, Seeland U, Rosenkranz S, Feiden W, Rube C, Rube CE: Matrix-metallo-proteinases and their tissue inhibitors in radiation-induced lung injury. Int J Radiat Biol 2007, 83:665-676

27. Chen P, McGuire JK, Hackman RC, Kim KH, Black RA, Poindexter K, Yan W, Liu P, Chen AJ, Parks WC, Madtes DK: Tissue inhibitor of metalloproteinase-1 moderates airway re-epithelialization by regulating matrilysin activity. Am J Pathol 2008, 172:1256-1270

28. Morrell NW: Role of bone morphogenetic protein receptors in the development of pulmonary arterial hypertension. Adv Exp Med Biol 661:251-264

29. Di Bernardo MC, Matakidou A, Eisen T, Houlston RS: Plasminogen activator inhibitor variants PAI-1 A15T and PAI-2 S413C influence lung cancer prognosis. Lung Cancer 2009, 65:237-241

30. Kucharewicz I, Mogielnicki A, Kasacka I, Buczko W, Bodzenta-Lukaszyk A: Plasmin system regulation in an ovalbumin-induced rat model of asthma. Int Arch Allergy Immunol 2008, 147:190-196

31. Yang YL, Liu YS, Chuang LY, Guh JY, Lee TC, Liao TN, Hung MY, Chiang TA: Bone morphogenetic protein-2 antagonizes renal interstitial fibrosis by promoting catabolism of type I transforming growth factor-beta receptors. Endocrinology 2009, 150:727-740

32. Myllarniemi M, Vuorinen K, Pulkkinen V, Kankaanranta $H$, Aine $T$, Salmenkivi K, Keski-Oja J, Koli K, Kinnula V: Gremlin localization and expression levels partially differentiate idiopathic interstitial pneumonia severity and subtype. J Pathol 2008, 214:456-463

33. Bock O, Hoftmann J, Theophile K, Hussein K, Wiese B, Schlue J, Kreipe $\mathrm{H}$ : Bone morphogenetic proteins are overexpressed in the bone marrow of primary myelofibrosis and are apparently induced by fibrogenic cytokines. Am J Pathol 2008, 172:951-960

34. Liu SB, Hu PZ, Hou Y, Li P, Cao W, Tian Q: Recombinant human bone morphogenetic protein-2 promotes the proliferation of mesenchymal stem cells in vivo and in vitro. Chin Med J (Engl) 2009, 122:839-843

35. Costello CM, Cahill E, Martin F, Gaine S, McLoughlin P: Role of gremlin in the lung: development and disease. Am J Respir Cell Mol Biol 42:517-523

36. Gosselink JV, Hayashi S, Elliott WM, Xing L, Chan B, Yang L, Wright C, Sin D, Pare PD, Pierce JA, Pierce RA, Patterson A, Cooper J, Hogg JC: Differential expression of tissue repair genes in the pathogenesis of COPD. Am J Respir Crit Care Med 2010, 181:1329-1335

37. Ide M, Ishii $H$, Mukae $H$, Iwata A, Sakamoto N, Kadota J, Kohno S: High serum levels of thrombospondin-1 in patients with idiopathic interstitial pneumonia. Respir Med 2008, 102:16251630

38. Crosby LM, Waters CM: Epithelial repair mechanisms in the lung. Am J Physiol Lung Cell Mol Physiol 2010, 298:L715-L731 